Journal of Nepal Mathematical Society (JNMS), Vol. 4, Issue 2 (2021); L. de O. Miranda, L. B. B. Miranda

\title{
One Solution for Many Linear Partial Differential Equations With Terms of Equal Orders
}

\author{
Lohans de Oliveira Miranda ${ }^{1}$, Lossian Barbosa Bacelar Miranda ${ }^{2}$ \\ ${ }^{1}$ Unisul University, Brazil \\ 2 IFPI, Brazil \\ Correspondence to: Lossian Barbosa Bacelar Miranda, Email: lossianm@gmail.com
}

\begin{abstract}
We disclose a simple and straightforward method of solving single-order linear partial differential equations. The advantage of the method is that it is applicable to any orders and the big disadvantage is that it is restricted to a single order at a time. As it is very easy compared to classical methods, it has didactic value.
\end{abstract}

Keywords: Single order linear partial differential equations, Laplacian equation, Harmonic polynomials, $k$-dimensional Hessian matrix

DOI: https://doi.org/10.3126/jnms.v4i2.41462

\section{Introduction}

A partial differential equation is called linear if it is linear in the unknown function and its derivatives. Using multi-index notation, a partial differential equation is said to be linear if it has the form $\sum_{|\alpha| \leq k} a_{\alpha}(x) D^{\alpha} u=$ $f(x)$ for given functions $a_{\alpha}(|\alpha| \leq k)$ and $f[3$. We do not need to work with multi-index notation as all terms (all of the same order) of the linear equations involved are products of functions by entries of the " $k$-dimensional Hessian matrix". We use a more simplified notation in which may occur $\partial x_{i_{p}}=\partial x_{i_{q}}$ in $\frac{\partial^{k} u}{\partial x_{i_{1}} \partial x_{i_{2}} \ldots \partial x_{i_{k}}}(\vec{x})$ with $p \neq q$. Here, we deal with linear partial differential equations written in the form of the equation (4) in the reference [1], taking $L_{1} u=0$. According to Florian Cajori [4], the first ideas related to partial derivation are from Leibniz, however, the perfect establishment of partial differential equations was made by Nicolaus Bernoulli : "Partial differential equations stand out clearly in six examples on trajectories published in 1719 by Nicolaus Bernoulli (1695 - 1726), the twenty-four year old son of John". With Euler and Cauchy, this branch of Mathematics almost acquired its current visibility, with Euler being a milestone in the study of partial differential equations. Very little is known about non-linear equations and, about linear equations, there is still no general theory that describes or even connects the various solving methods developed since Euler. Here, we present one more method. However, as the method presented is very simple, applicable to any order and based on an additive philosophical principle, it can be a significant milestone for the linear equations. Many important partial differential equations such as of Poisson, Laplace, Wave and Tricomi can be studied using the methods and results set out here. We make very strong restrictions on the functions $h_{s}\left(\bar{x}_{i_{s}}\right)(w=1,2, \ldots, s-1)$ and $h_{w}\left(\bar{x}_{i_{w}}\right)$ obtained from the integrations with application of the fundamental theorem of calculus. More detailed research may use less restrictive conditions to get more refined results. The Proposition 4 of this scientific article points towards an alternative way of studying harmonic functions.

\section{Preliminaries}

Let us Consider:

1. $\vec{x}=\left(x_{1}, x_{2}, \ldots, x_{n}\right) \in A, A$ open set in $R^{n}$;

2. $k \in I_{n}=\{1,2,3, \ldots, n\}$; 
3. $u: A \rightarrow \mathbb{R}$, differentiable function ( $k$ times), with continuous derivatives.

Consider the $k$-dimensional Hessian matrix:

$$
H=\left(\frac{\partial^{k} u}{\partial x_{i_{1}} \partial x_{i_{2}} \ldots \partial x_{i_{k}}}(\vec{x})\right)
$$

From $H$, let us consider the following system, being $b_{i_{1} i_{2} \ldots i_{k}}(\vec{x})$ and $f_{i_{1} i_{2} \ldots i_{k}}(\vec{x})$ differentiable functions of order $k$, with continuous derivatives, and $\frac{f_{i_{1} i_{2} \ldots i_{k}}(\vec{x})}{b_{i_{1} i_{2} \ldots i_{k}}(\vec{x})}$ being well defined in $A$ :

$$
\left(b_{i_{1} i_{2} \ldots i_{k}}(\vec{x}) \frac{\partial^{k} u}{\partial x_{i_{1}} \partial x_{i_{2}} \ldots \partial x_{i_{k}}}(\vec{x})\right)=f_{i_{1} i_{2} \ldots i_{k}}(\vec{x})
$$

Let us denote

$$
g_{i_{1} i_{2} \ldots i_{k}}(\vec{x})=\frac{f_{i_{1} i_{2} \ldots i_{k}}(\vec{x})}{b_{i_{1} i_{2} \ldots i_{k}}(\vec{x})}
$$

So relation 2 can be written as

$$
\left(\frac{\partial^{k} u}{\partial x_{i_{1}} \partial x_{i_{2}} \ldots \partial x_{i_{k}}}(\vec{x})\right)=\left(g_{i_{1} i_{2} \ldots i_{k}}(\vec{x})\right)
$$

Observation 1. Repeated applications of the fundamental theorem of calculus for each of the $n^{k}$ partial differential equations

$$
\frac{\partial^{k} u}{\partial x_{i_{1}} \partial x_{i_{2}} \ldots \partial x_{i_{k}}}(\vec{x})=g_{i_{1} i_{2} \ldots i_{k}}(\vec{x})
$$

give us the $n^{k}$ solutions 1

$$
u_{i_{1} i_{2} \ldots i_{k}}(\vec{x})=\iiint \ldots \int g_{i_{1} i_{2} \ldots i_{k}}(\vec{x}) d x_{i_{1}} d x_{i_{2}} \ldots d x_{i_{k-1}}+\sum_{s=1}^{k-1}\left(c_{s, i_{1} i_{2} \ldots i_{k}} \prod_{\theta=s+1}^{k-1} x_{i_{\theta}}\right)+c_{k-1, i_{1} i_{2} \ldots i_{k}} .
$$

Here, the $c$ (under indexed) are real or complex numbers. Obviously,

$$
\frac{\partial^{k} u_{i_{1} i_{2} \ldots i_{k}}}{\partial x_{j_{1}} \partial x_{j_{2}} \ldots \partial x_{j_{k}}}(\vec{x})=g_{i_{1} i_{2} \ldots i_{k}}(\vec{x}), \text { if }\left(i_{1}, i_{2}, \ldots, i_{k}\right)=\left(j_{1}, j_{2}, \ldots, j_{k}\right) .
$$

Consider the function

$$
\tilde{u}(\vec{x})=\sum_{i_{1}, i_{2}, \ldots, i_{k} \in I_{n}} u_{i_{1} i_{2} \ldots i_{k}}(\vec{x})
$$

Now, we can state the main result.

${ }^{1}$ Here, as in [7, we will take $n>1$, since the case $n=1$ is special [6]. By induction in relation to $s$ we prove that
$\frac{\partial^{k-s} u}{\partial x_{i_{s+1}} \partial x_{i_{s+2}} \ldots \partial x_{i_{k}}}(\vec{x})=\iiint \ldots \int g_{i_{1} i_{2} \ldots i_{k}}(\vec{x}) d x_{i_{1}} d x_{i_{2}} \ldots d x_{i_{s}}+\sum_{w=1}^{s-1}\left(\iint \ldots \int h_{w}\left(\bar{x}_{i_{w}}\right) d x_{i_{w+1}} d x_{i_{w+2}} \ldots d x_{i_{s}}\right)+h_{s}\left(\bar{x}_{i_{s}}\right), \forall s \in$ $N, 1 \leq s \leq k$. The functions $h_{s}\left(\bar{x}_{i_{s}}\right)(w=1,2, \ldots, s-1)$ and $h_{w}\left(\bar{x}_{i_{w}}\right)$ are any differentiable functions ( $k$ times), with continuous derivatives, which do not depend on $\bar{x}_{i_{s}}$ and $\bar{x}_{i_{w}}$ respectively. They are obtained directly from the integration via the fundamental theorem of calculus. Then choosing $s=k$ and assuming that $h_{w}\left(\bar{x}_{i_{w}}\right)=h_{w}$ and $h_{k}\left(\bar{x}_{i_{k}}\right)=h_{k}$ are constant functions, we will have the solution $u(\vec{x})=u_{i_{1} i_{2} \ldots i_{k}}(\vec{x})=\iiint \ldots \int g_{i_{1} i_{2} \ldots i_{k}}(\vec{x}) d x_{i_{1}} d x_{i_{2}} \ldots d x_{i_{k}}+\sum_{w=1}^{k-1}\left(h_{w} x_{i_{w+1}} x_{i_{w+2}} \ldots x_{i_{k}}\right)+$ $h_{k}$. Note that we can take $h_{w}$ to be any real number, for any $w, 0<w<k+1$. Using the notations $h_{w}=c_{s, i_{1} i_{2} \ldots i_{k}}$, $h_{k}=c_{k-1, i_{1} i_{2} \ldots i_{k}}$ and $x_{i_{w+1}} x_{i_{w+2}} \ldots x_{i_{k}}=\prod_{\theta=s+1}^{k-1} x_{i_{\theta}}$, we will have the Equation [6. 


\section{Main results}

Proposition 1. In the hypotheses established above, if for $\left(i_{1}, i_{2}, \ldots, i_{k}\right) \neq\left(j_{1}, j_{2}, \ldots, j_{k}\right)$, we have,

$$
\frac{\partial^{k} u_{i_{1} i_{2} \ldots i_{k}}}{\partial x_{j_{1}} \partial x_{j_{2} \ldots \partial x_{j_{k}}}}(\vec{x})=0,
$$

then $\tilde{u}(\vec{x})=\sum_{i_{1}, i_{2}, \ldots, i_{k} \in I_{n}} u_{i_{1} i_{2} \ldots i_{k}}(\vec{x})$ defined in (8) will be the solution of $n^{k}$ partial differential equations defined in (2), or alternatively in (5). In particular, $\tilde{u}(\vec{x})$ will be a solution of the $2^{n^{k}}-1$ differential equations defined by the sums of the elements of all non-empty subsets of the set

$$
B=\left\{\frac{\partial^{k} u}{\partial x_{i_{1}} \partial x_{i_{2}} \ldots \partial x_{i_{k}}}(\vec{x}) ; i_{1}, i_{2}, \ldots, i_{k} \in I_{n}\right\}
$$

Demonstration. This proposition is an immediate consequence of the construction of $\tilde{u}(\vec{x})$ and of the hypothesis $\left(i_{1}, i_{2}, \ldots, i_{k}\right) \neq\left(j_{1}, j_{2}, \ldots, j_{k}\right)$.

Observation 2. The thesis ${ }^{2}$ of Proposition 1 can still be obtained even if the assumptions established in (9) are not satisfied. To do so, it is enough to find the unknown functions involved that satisfy the required integral equations.

Proposition 2. Let $f: R^{2} \mapsto R$ analytic function. So, if $f$ is an affine function, then

$$
L(f)(x, y)=\iint \frac{\partial^{2} f}{\partial y^{2}}(x, y) d x d x-\iint \frac{\partial^{2} f}{\partial x^{2}}(x, y) d y d y=0 .
$$

Demonstration. If $f(x, y)=a x+b y+c$, then $\frac{\partial^{2} f}{\partial y^{2}}=\frac{\partial^{2} f}{\partial x^{2}}$ and from that follows 11.

\section{Applications}

When considering order 2, the above method solves the main science equations such as the Wave, Laplace and Poisson equations. In particular, they give the most obvious solutions in the case of the Wave equation, such as simple vibrating string translations. In the case of the Poisson equation, they give very varied solutions. Let's look at a case of Laplace's equation.

\subsection{Laplacian equation}

$$
\left(\begin{array}{cc}
\frac{\partial^{2} u}{\partial x^{2}} & 0 \\
0 & \frac{\partial^{2} u}{\partial y^{2}}
\end{array}\right)=\left(\begin{array}{cc}
f(x, y) & 0 \\
0 & -f(x, y)
\end{array}\right)
$$

By the presented theory, we have

$$
\begin{gathered}
u_{11}(x, y)=\iint f(x, y) d x d x+k_{1}(y) x+k_{2}(y) \\
u_{22}(x, y)=-\iint f(x, y) d y d y+v_{1}(x) y+v_{2}(x) \\
\tilde{u}(x, y)=\iint f(x, y) d x d x-\iint f(x, y) d y d y+k_{1}(y) x+k_{2}(y)+v_{1}(x) y+v_{2}(x)
\end{gathered}
$$

The hypotheses established in $(9)$ are not satisfied because, for example,

$$
\frac{\partial^{2} u_{11}}{\partial y^{2}}(x, y)=\iint \frac{\partial^{2} f}{\partial y^{2}}(x, y) d x d x+k_{1}^{\prime \prime}(y) x+k_{2}^{\prime \prime}(y) .
$$

\footnotetext{
${ }^{2}$ A Theorem is a Proposition which places before us some truth which it is proposed to demonstrate. In the enunciation of a Theorem, there are always two parts, called the 'Hypothesis,' and the 'Thesis,' or 'Conclusion' [2].
} 
But if we take $k_{1}(y), k_{2}(y), v_{1}(x)$ and $v_{2}(x)$ to be affine functions, all their second derivatives will be nullified and we will obtain from (13) the candidate for harmonic function, renamed with the same nomenclature, as follows

$$
\tilde{u}(x, y)=\iint f(x, y) d x d x-\iint f(x, y) d y d y+a x y+b x+c y+d
$$

In (14), the parameters are any real or complex numbers. Derived (14) we will have

$$
\frac{\partial^{2} \tilde{u}}{\partial x^{2}}(x, y)+\frac{\partial^{2} \tilde{u}}{\partial y^{2}}(x, y)=\left(f(x, y)-\iint \frac{\partial^{2} f}{\partial x^{2}}(x, y) d y d y\right)+\left(\iint \frac{\partial^{2} f}{\partial y^{2}}(x, y) d x d x-f(x, y)\right)
$$

This last sum, equaled to zero, gives us

$$
\iint \frac{\partial^{2} f}{\partial y^{2}}(x, y) d x d x-\iint \frac{\partial^{2} f}{\partial x^{2}}(x, y) d y d y=0 .
$$

By Proposition 2, $\tilde{u}(x, y)$ in (14) will be harmonic (solution of Laplace's equation) if $f(x, y)$ is an affine function, that is of type

$$
f(x, y)=A x+B y+D
$$

Substituting 15 into 14 gives the three-degree harmonic polynomial of the form

$$
\tilde{u}(x, y)=\frac{A}{2}\left(\frac{x^{3}}{3}-x y^{2}\right)+\frac{B}{2}\left(x^{2} y-\frac{y^{3}}{3}\right)+\frac{D}{2}\left(x^{2}-y^{2}\right)+a x y+b x+c y+d
$$

Proposition 3. The function

$$
\tilde{u}(x, y)=\iint f(x, y) d x d x-\iint f(x, y) d y d y+a x y+b x+c y+d
$$

will be harmonic if and only if $f(x, y)$ belongs to the kernel of the linear transformation

$$
L(f)(x, y)=\iint \frac{\partial^{2} f}{\partial y^{2}}(x, y) d x d x-\iint \frac{\partial^{2} f}{\partial x^{2}}(x, y) d y d y .
$$

Moreover, if $\frac{\partial^{2} f}{\partial y^{2}}-\frac{\partial^{2} f}{\partial x^{2}}=0$, then $f$ will belong to that same kernel.

\section{Demonstration.}

$(\Rightarrow)$ :

$$
0=\iint\left(\frac{\partial^{2} f}{\partial y^{2}}+\frac{\partial^{2} f}{\partial x^{2}}\right) d x d x-\iint\left(\frac{\partial^{2} f}{\partial y^{2}}+\frac{\partial^{2} f}{\partial x^{2}}\right) d y d y=L(f)(x, y)
$$

$(\Leftarrow)$ : Conversely, we have $\Delta(\tilde{u})(x, y)=L(f)(x, y)$. By hypothesis, the latter is the null function. From $\frac{\partial^{2} f}{\partial y^{2}}=\frac{\partial^{2} f}{\partial x^{2}}$ follow, by simple derivation,

$$
\iint \frac{\partial^{2} f}{\partial y^{2}}(x, y) d x d x=\iint \frac{\partial^{2} f}{\partial x^{2}}(x, y) d x d x=f(x, y)
$$

and

$$
\iint \frac{\partial^{2} f}{\partial y^{2}}(x, y) d y d y=\iint \frac{\partial^{2} f}{\partial x^{2}}(x, y) d y d y=f(x, y) .
$$

And, from these results $L(f)(x, y)=0$.

Proposition 4. The function

$$
H(x, y)=\sum_{n=0}^{\infty}\left(\sum_{i=0}^{n} h_{i, n-i} x^{i} y^{n-i}\right)
$$



is analytic harmonic if and only if exists analytic function

$$
f(x, y)=\sum_{n=0}^{\infty}\left(\sum_{i=0}^{n} a_{i, n-i} x^{i} y^{n-i}\right)
$$

and numbers $a, b, c, d$ such that

$$
H(x, y)=\iint f(x, y) d x d x-\iint f(x, y) d y d y+a x y+b x+c y+d
$$

and $f(x, y)$ belong to the kernel of the linear transformation

$$
L(f)(x, y)=\iint \frac{\partial^{2} f}{\partial y^{2}}(x, y) d x d x-\iint \frac{\partial^{2} f}{\partial x^{2}}(x, y) d y d y .
$$

Demonstration. $(\Rightarrow)$ : If $H(x, y)$ is an affine function, let's say

$$
H(x, y)=a x+b y+c,
$$

let's take $f(x, y)=0$. Then

$$
H(x, y)=a x+b y+c=\iint 0 d x d x-\iint 0 d y d y+a x+b y+c .
$$

Let us consider the case where $H(x, y)$ is not an affine function. We will first prove that if $f$ exists, then it belongs to $\operatorname{Ker}(L)$ (kernel of $L)$. Let

$$
H(x, y)=\iint f(x, y) d x d x-\iint f(x, y) d y d y+a x y+b x+c y+d
$$

. Then,

$$
\left.0=\Delta H(x, y)=\Delta\left(\iint f(x, y) d x d x-\iint f(x, y) d y d y+a x y+b x+c y+d\right)=L(f)(x, y)\right) .
$$

Let's prove that $f(x, y)$ exists. We have

$$
H(x, y)=\frac{H(x, y)}{2}+\frac{H(x, y)}{2}=\frac{H(x, y)}{2}-\frac{1}{2} \iint\left(-\frac{\partial^{2} H}{\partial y^{2}}\right)(x, y) d y d y
$$

Since, by hypothesis,

$$
-\frac{\partial^{2} H}{\partial y^{2}}(x, y)=\frac{\partial^{2} H}{\partial x^{2}}(x, y)
$$

we have,

$H(x, y)=\frac{1}{2} \iint \frac{\partial^{2} H}{\partial x^{2}}(x, y) d x d x-\frac{1}{2} \iint \frac{\partial^{2} H}{\partial x^{2}}(x, y) d y d y=\iint \frac{1}{2} \frac{\partial^{2} H}{\partial x^{2}}(x, y) d x d x-\iint \frac{1}{2} \frac{\partial^{2} H}{\partial x^{2}}(x, y) d y d y$.

So there exists $f(x, y)=\frac{1}{2} \frac{\partial^{2} H}{\partial x^{2}}(x, y)$.

$(\Leftarrow):$

$$
\Delta H=\Delta\left(\iint f(x, y) d x d x-\iint f(x, y) d y d y+a x y+b x+c y+d\right)=L(f)=0 .
$$

Then, $H(x, y)$ is the harmonic analytic function.

Observation 3. In relation to Proposition 4, note that

$$
\Delta H=2 a_{20}+2 a_{02}+\sum_{n=3}^{\infty}\left(\sum_{i=0}^{n} h_{i, n-i}\left(i(i-1) x^{i-2} y^{n-i}+(n-i)(n-i-1) x^{i} y^{n-i-2}\right)\right)
$$


One solution for many linear partial differential equations with terms of equal orders

$L(f)(x, y)=a_{02} x^{2}-a_{20} y^{2}+\sum_{n=3}^{\infty}\left(\sum_{i=0}^{n} a_{i, n-i}\left(\frac{(n-i)(n-i-1)}{(i+2)(i+1)} x^{i+2} y^{n-i-2}-\frac{i(i-1)}{(n-i+2)(n-i+1)} x^{i-2} y^{n-i+2}\right)\right)$

Note that, for

$$
f(x, y)=e^{-x} \text { siny, } L(f)(x, y)=0
$$

but

$$
\frac{\partial^{2} f}{\partial y^{2}}(x, y)-\frac{\partial^{2} f}{\partial x^{2}}(x, y)=-2 e^{-x} \sin y \neq 0
$$

See that

$$
\iint f(x, y) d x d x=\iint f(x, y) d y d y \Leftrightarrow \frac{\partial^{2} f}{\partial y^{2}}(x, y)=\frac{\partial^{2} f}{\partial x^{2}}(x, y) .
$$

Solving $L(f)(x, y)=0$ considering $f(x, y)=g(x) h(y)$, we get

$$
f(x, y)=\left(c_{1} e^{-k x}+c_{2} e^{k x}+c_{3} \sin k x+c_{4} \cos k x\right)\left(d_{1} e^{-k y}+d_{2} e^{k y}+d_{3} \sin k y+d_{4} \cos k y\right)
$$

The same equation can be solved by taking the series

$$
f(x, y)=\sum_{n=0}^{\infty}\left(\sum_{i=0}^{n} a_{i, n-i} x^{i} y^{n-1}\right)
$$

The null polynomial of degree $n$ obtained in $L(f)(x, y)=0$ is

$$
\begin{gathered}
\sum_{i=0}^{n-4}\left(\frac{(n-i)(n-i-1)}{(2+i)(1+i)} a_{i, n-i}-\frac{(i+4)(i+3)}{(i+6)(i+5)} a_{i+4, n-i-4}\right) x^{2+i} y^{n-2-i}+\frac{2}{n(n-1)} a_{n-2,2} x^{8} \\
-\frac{2}{n(n-1)} a_{2, n-2} y^{8}+\frac{3 \times 2}{(n-1)(n-2)} a_{n-3,3} x^{n-1} y-\frac{3 \times 2}{(n-1)(n-2)} a_{3, n-3} x y^{n-1}
\end{gathered}
$$

This identity provides

$$
a_{i+4, n-i-4}=\frac{(i+6)(i+5)(n-i)(n-i-1)}{(i+4)(i+3)(i+2)(i+1)} a_{i, n-i}
$$

The entire problem is conditioned to convergences for the coefficients chosen with these restrictions. In particular, all coefficients $a_{i, n-i}$ can be placed according to the coefficients of the types $a_{0, k}, a_{1, k} ; k \in N$. Note that on average, five-ninths of the coefficients $a_{i, n-i}$ are obligatorily null. And those that are not necessarily null are all of the types $a_{4 i, 4 k-4 i} x^{4 i} y^{4 k-4 i}, a_{4 i, 4 k+1-4 i} x^{4 i} y^{4 k+1-4 i}, a_{4 i+1,4 k-4 i} x^{4 i+1} y^{4 k-4 i}$ and $a_{4 i+1,4 k+1-4 i} x^{4 i+1} y^{4 k+1-4 i}$, with $n=4 k, 0 \leq i \leq k \in N$.

\section{Conclusion}

Classical methods such as those presented in [5] encompass the method under study. However, this is explicit in many situations. Furthermore, the mathematical tools needed to understand the method are restricted to the most basic differential calculus. An approach such as the one established would be of great encouragement for students in their first contact with the theory of partial differential equations. And to further facilitate learning, we could develop the results initially in dimensions two and three. The linear transformation $L(f)(x, y)$ establishes an alternative method for the famous Laplacian operator. Analogous situations must occur for many other operators. The analytic functions that belong to the kernel of $L$ seem to be simpler than the harmonic functions.

\section{References}

[1] Encyclopedia of Mathematics, 2001, Differential equation, partial, EMS Press. 
Journal of Nepal Mathematical Society (JNMS), Vol. 4, Issue 2 (2021); L. de O. Miranda, L. B. B. Miranda

[2] Euclides, 1884, An introduction to geometry, consisting of Euclid's Elements, Book I, accompanied by numerous explanations, questions, and exercises, by J. Walmsley. [With] Answers, Volume 1, Francis Hodgson, London.

[3] Evans, Lawrence C., 2010, Partial Differential Equations, Second Edition, AMS, Providence, Rhode Island, 2010.

[4] Florian Cajori, 1928, The early history of partial differential equations and of partial differentiation and integration, The American Mathematical Monthly, Vol. 35 (9), 459-467.

[5] Gerald B. Folland, 1995, Introduction to partial differential equations, Second Edition, Princeton University Press, New Jersey.

[6] Lima, Elon Lages, 1992, Curso de Análise, Vol. 1, SBM, Rio de Janeiro, 1992.

[7] Sheldon Axler, Paul Bourdon, and Wade Ramey, 2001, Harmonic function theory, Second Edition, Springer-Verlag, New York. 